Lewin, R. A. (1952). J. gen. Microbiol. 6, 233-248.

\title{
Ultraviolet Induced Mutations in Chlamydomonas moewusii Gerloff
}

\author{
By R. A. LEWIN \\ Osborn Botanical Laboratory, Yale University, New Haven, Conn., U.S.A.
}

\begin{abstract}
SUMMARY: Mutations were induced in the unicellular green alga Chlamydomonas moewusii Gerloff by ultraviolet irradiation. The majority of mutants detected were slow-growing types which did not respond to organic supplements in the medium, and palmelloid forms in which flagellated cells were not produced, or only occurred in small numbers. Among biochemical mutants, one exhibited impairment of the photosynthetic mechanism, and two were vitamin-dependent, respectively requiring $p$-aminobenzoic acid (PABA) and aneurin for growth. Some mutants were distinguished by features of cell contents, cell form, or mode of cell division, while in others motility of the cells was restricted by the absence or paralysis of the flagella.
\end{abstract}

Though it is of some importance to investigate physiological and genetical processes in green plants by the use of a micro-organism with a comparatively short life cycle, there have been very few genetic studies carried out with algae. The bulk of the literature in this limited field is the work of F. Moewus, and his reported results, almost without exception, have remained unconfirmed.

Mating types of Chlamydomonas moerousii were isolated by Dr L. Provasoli in 1948, and the physiology and life cycle of this organism have been studied (Lewin, J. C., 1950; Hutner \& Provasoli, 1951; Lewin, R. A., 1952a). The organism is completely-and, it seems, obligately-autotrophic, grows well on mineral agar media, and in suitable conditions the cell number will double in $12 \mathrm{hr}$. The vegetative cells are haploid. Gamete formation can be readily induced, and zygote formation and germination may be controlled experimentally (Lewin, R. A., 1949). Meiosis takes place within the zygospore during germination; the haploid cells which emerge can be separated (Moewus, 1940a), and from these cells new vegetative clones can be established.

The species is regularly heterothallic: the mating-type character segregates in the expected 2:2 or 4:4 fashion, as in Dunaliella salina (Lerche, 1937) and C. Reinhardi (Smith \& Regnery, 1950). Since the two original clones of complementary mating-type (here designated plus and minus, or WT + and WT -) appear identical except for mating behaviour (Lewin, R. A., 1950a), it was necessary to obtain other marker genes in order to examine the genetics of the organism. The genetic data are presented elsewhere (Lewin, R. A., 1952b).

Lerche (1937) found that $D$. salina oblonga, which she had isolated from natural sources, differed from $D$. salina salina in a single gene. In $C$. moewusii, the frequency of recognized spontaneous mutation appears to be fairly low. (In one experiment, among $c .1200$ colonies examined only eight were found which in size or contour differed in any way from the rest. Of these, two were persistently aberrant in subculture: one produced slightly drier colonies than 
normal, while the other-which subsequently reverted twice to the wild-typegrew slowly as a palmella on all media.) Henceforth in this paper only 'induced' mutations will be considered. Proof of the experimental induction of any given mutant cannot be presented, and the epithet 'induced' is used loosely, to cover all changes detected after irradiation-i.e. post hoc, not necessarily propter hoc.

Gerloff (1940) attempted to induce mutations in C. terricola and C. humophilos by ultraviolet light. He used a Hanau ultraviolet source (S.N. 300) at a distance of $35 \mathrm{~cm}$. for durations between 20 sec. and $5 \mathrm{~min}$., but was unable to detect any morphological or sexually aberrant mutants. After X-irradiation dosages of 1500,3000 and 6000 r. of vegetative cells and zygotes, he detected two variants of the latter species, characterized by frequent reduplication of the pyrenoid. This character, however, was not sustained in the progeny after sexual reproduction, and may have been attributable to cytoplasmic disturbance.

Moewus (1940b) reported that, by subjecting cells of $C$. eugametos to treatments of heat shock, appreciable numbers of mutant types could be obtained. Thus when cell suspensions were heated to $75^{\circ}$ for 15 min., about $25 \%$ of the cells were killed, and among the survivors each of certain loci concerned with sexual behaviour exhibited a mutation frequency of $c .0 \cdot 2-0 \cdot 3 \%$. Vegetative cells of $C$. moewusii do not survive heating to $60^{\circ}$ for 30 sec., and no attempt was made by the present author to induce mutations in this species by heatshock.

Of several possible mutagenic agents considered, ultraviolet was deemed the most suitable, for the following reasons: (1) The use of radiation rather than chemical mutagens obviates the necessity of subsequent washing and the dangers of persistent contamination. (2) There is good evidence that, in most organisms studied, ultraviolet effects are mainly 'point' mutations, while $\mathrm{X}$-rays tend predominantly to induce gross chromosome damage (Lea, 1947). Changes of the latter sort were not desired in these preliminary investigations, since they might complicate genetic analyses. (3) An ultraviolet lamp is a convenient laboratory tool, easily handled, and occupying small space.

\section{METHODS}

Cultures were all grown in continuous light from batteries of 'daylight' fluorescent tubes, at an intensity of $c .470$ f.c. and at a constant temperature of $23^{\circ}$. The media employed are given in Table 1. Further details of general methods employed in these investigations are published elsewhere (Lewin, R. A., 1952a).

\section{Detection of mutant types: preliminary considerations}

A Westinghouse 'Sterilamp' (model WL-782-30) was employed: the lamp was turned on $15-30 \mathrm{~min}$. before use.

For all experiments in mutagenesis, in which cell aggregates are obviously undesirable, free-cell suspensions were used from young cultures grown in 
media of $\mathrm{pH} 7.0$ or somewhat higher. In more acid media, the cells tend to form palmelloid colonies, in which most mutants would be overgrown by the associated parental-type cells.

A modification of the 'layering' technique evolved by Lederberg \& Tatum (1946) for the detection of mutants was initially employed. Although in two extensive experiments a total of fifteen mutants was obtained (among them M. 151-see p. 246), use of this method was discontinued for the following

\section{Table 1. Composition of culture media}

BM

Beijerinck's mineral medium

$\begin{array}{ll}\mathrm{NH}_{4} \mathrm{NO}_{3} & 0.5 \mathrm{~g} . / 1 . \\ \mathrm{K}_{2} \mathrm{HPO}_{4} & 0.2 \mathrm{~g} . / 1 . \\ \mathrm{MgSO}_{4} \cdot 7 \mathrm{H}_{2} \mathrm{O} & 0.2 \mathrm{~g} \cdot / \mathrm{l} . \\ \mathrm{CaCl}_{2} \cdot 2 \mathrm{H}_{2} \mathrm{O} & 0.1 \mathrm{~g} . / \mathrm{l} .\end{array}$

Trace elements (Burkholder \& Nickell, 1949)

BPM More strongly buffered, as BM but with phosphate at triple strength $-0 \cdot 6 \mathrm{~g} . / 1$.

BA As BM, supplemented with $0 \cdot 1 \mathrm{~g}$. Na acetate.

BAT As BA, supplemented with 0.4 g. Bacto-tryptone (Difco).

BATY As BAT, supplemented with $0 \cdot 1 \mathrm{~g}$. yeast extract (Difco).

reasons: (1) Irradiation in a liquid medium results in a dosage per cell which is statistically constant, but not individually uniform. Some cells may by chance receive higher dosages, while others, perhaps in a backwater of the vessel and shielded on one side by the glass wall, escape with a relatively light dose. The latter will be well represented among the surviving cells which give rise to colonies. (2) The incorporation of $C$. moerousii into liquid agar necessitates some care, since the cells are killed at temperatures approaching that at which the medium solidifies. All vegetative cells are killed by exposure to $48^{\circ}$ for $1 \mathrm{~min}$. or to $46^{\circ}$ for $1 \frac{1}{2} \mathrm{~min}$. It is certain that many cells succumb at temperatures lower than these, and this sensitivity will be a function of the physiological state of the cells (and thus presumably of the ultraviolet dosage sustained). (3) The need for three or four layers of agar multiplies the chance of invasion by aerial contaminants. This is particularly serious in the case of an organism with a generation time of several hours, where 10 days are required for the completion of the layering process. Moreover, the removal of suspected mutant colonies from the depths of the agar involves possible contamination from neighbouring colonies which, despite the precaution of 'sandwiching', may yet invade agar/agar interfaces. (4) In this preliminary study, morphological and various other mutant types were considered, though they grow well in mineral media, and would therefore be overlooked by the adoption of this method. (5) The rate of growth of all colonies is considerably reduced by the limiting rate of diffusion of carbon dioxide down to the embedded cells.

For these reasons, as well as for general considerations of ease and convenience, a simpler method was adopted, which actually gave better results. About $65 \%$ of the putative mutant colonies obtained by the layering technique proved on subsequent testing to be indistinguishable from the parent type-colony 
development having followed an initial lag probably attributable to ultraviolet damage in the non-hereditary soma. In the following method, this fraction was reduced to about $50 \%$.

\section{Surface irradiation}

About $\mathbf{0} \cdot 1 \mathrm{ml}$. of a dense suspension of actively growing cells was pipetted on the surface of a minimal (BM) agar plate, containing a non-inhibitory concentration of an indicator dye (see p. 237). By means of a sterilized spreader (a glass rod, so bent that a straight section 5-8 $\mathrm{cm}$. in length can be applied to the surface of the agar and stroked over it without breaking the surface), the suspension was dispersed as evenly as possible over the surface, and allowed to dry. The plates were then irradiated, open, for varying periods of time (0-5 min. at $10 \mathrm{~cm}$. from the 'Sterilamp'), after which the covers were replaced, and the plates illuminated in the usual way without further treatment.

At the end of 5-7 days the colonies were scanned under the dissecting microscope, and all small, rough, or otherwise distinguishable colony types picked for further testing. (Plates too crowded, having received insufficient irradiation to reduce the surface population, were discarded.) As mentioned above, about half the variants so isolated proved to remain constant in subculture.

It was of interest to know the approximate percentage of cells surviving treatment, and to relate this to the proportion of mutants detected. For this reason, control (not irradiated) plates were prepared at the same time, and estimates of the numbers of viable (colony-producing) cells or cell aggregates were obtained by colony counts in random optical fields under the dissecting microscope, with which corresponding figures obtained from treated plates could be compared.

It will be noted that, by this method, the following advantages were achieved: (1) Each cell received, within the physical limitations of the system, an identical dose of ultraviolet irradiation, which had not passed through a variable depth of aqueous cell suspension before reaching it. Cells near and partially shielded by the walls of the plate remained in this position, and high marginal survivals could be recognized and discounted if necessary. (2) The cells were at no time subjected to the unfavourable temperature of molten agar. (3) The resultant colonies were superficial and co-planar, considerably facilitating the isolation of single clones, and reducing the danger of crosscontamination. (4) Morphological colony variants could be detected. (5) The plates were only opened once between irradiation and 'picking'. (6) The diffusion path of $\mathrm{CO}_{2}$ from atmosphere to cell was reduced to a minimum.

The rationale of this method, where biochemical mutants are concerned, depends considerably on the size of the organism. The cell volume of $C$. moerwusii is approximately 100 times that of a cell of Bacterium coli, for which the layering technique was evolved. A cell, grown in a favourable medium and irradiated without special preliminary starvation or washing, may carry with it, in its cytoplasm or adsorbed on the wall, many times the minimum requirements of such nutrilites as it may require for growth. If the genetic ability to synthesize 
one such nutrilite is lost, growth ceases in the absence of an exogenous supply after a few cell generations. In the case of a bacterium, the resulting colony may be invisible under low magnification: in Chlamydomonas, however, it has proved easy to observe, remove, and subculture colonies estimated to contain 32-64 cells. Among colonies of this type, it may be expected-and in two cases (M. 336, M. 701) it was actually found-that nutritionally deficient types might be picked up.

Since well-characterized mutants might arise, differing from WT in their $\mathrm{pH}$ relations (e.g. due to an inability to utilize the ammonium ion as a nitrogen source), an indicator dye was incorporated into the medium. Preliminary experiments had shown that $10 \mathrm{mg}$./l. of bromthymol blue, or $40 \mathrm{mg}$./1. of neutral red, approached the concentrations at which growth was inhibited, though these dilutions had no appreciable effect. Actually, the result achieved was somewhat different from that originally anticipated. Local $\mathrm{pH}$ changes were not made apparent in the medium. On the other hand, colonies in which for some reason the cells were dying could readily be distinguished by the fact that the dead cells accumulated the dye to an appreciable degree. Thus M. 255, the growth rate of which does not differ from that of WT, was revealed by its greater sensitivity to low $\mathrm{pH}$. As the acidity of the medium (BM) rose with growth of the colonies, cells of this mutant died and took up the bromthymol blue (used in this case), so that the colony appeared orange-yellow, whereas its neighbours remained green. In most of such colonies there remained a sufficient number of viable cells to survive subculture (e.g. in one experiment, all but ten out of forty-six colonies containing dying cells were successfully transferred to complete medium and grown up).

Somewhat better results were obtained with neutral red than with bromthymol blue plates (see Table 2).

Table 2. Results of ultraviolet surface irradiation method for the detection of mutants

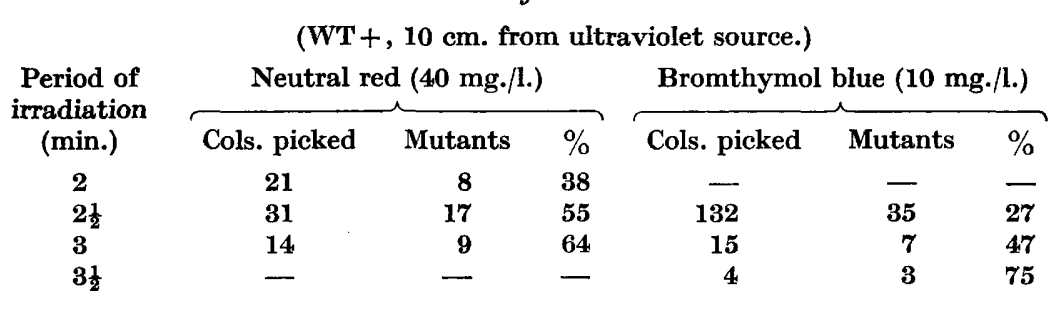

Screening of putative mutants

Colonies which were suspected of representing mutant types were assigned a serial number (M. 000), transferred to agar slants of a complete medium, smeared over the surface, and grown up in the light. Since, to many mutants of Chlorella, light proved to be lethal (Granick, S. Private communication), it might have been preferable, if possible, to grow these cultures in darkness. The possibility may also be envisaged that, in the illumination following ultraviolet irradiation, some mutations may be reversed and thereby lost; or, 
conversely, that a lethal effect associated with a mutation might be alleviated, thus enabling the mutant to survive. Such theoretical considerations must remain closed to practical investigations until the problem of obligate autotrophy in C. moerousii is solved.

The exact composition of the 'complete' medium was varied in the earlier series, but later only BATY was used. The advantages of using a solid, rather than a liquid, medium at this stage were twofold: (1) The slope could be conveniently handled and stored without further transfer. (2) Cells to be used as inoculum in biochemical studies could be scraped from the surface with a minimum of associated nutrilites as 'carry-over'.

A suspension of each such culture was then made in $\mathbf{0 . 1} \mathrm{ml}$. of sterile water, and from this tube loopfuls were spread on agar test media (Perkins, 1949). Three media were employed-BPM, BA, and BATY. The use of BA in addition to a minimal (i.e. purely mineral), and a complete medium, served to distinguish at once non-photosynthetic mutants from those with other growthfactor requirements, and, in the case of the latter, provided a check for the growth on minimal.

Eleven clones were tested on each set of three test plates, using a standard pattern grid on which the plates were set before inoculation. The plates were oriented by a scratched recognition mark at the edge, and a key to the positions recorded in the protocol. On each plate, the twelfth place was occupied by a control, of the parental type which had been subjected to irradiation.

After growth had proceeded for 5-7 days, the plates were examined, and any types deviant from the parent stock on any medium were marked for further investigation. Macroscopic comparison of growth and colony form was invariably supplemented by microscopic examination of cells from the 'complete' plate, and in many cases also from the other media. In this way a number of morphological, as well as some biochemical, mutants were detected.

Finally, a transfer was made from the minimal plate into a tube of liquid BPM, in order to expose any biochemically deficient mutants which might have grown using the traces of nutrient carried over from the original 'complete' slope, or present in the agar. Several non-swimming mutants were also revealed in this way.

\section{TYPES OF MUTANTS OBTAINED}

Over half of the colonies tested behaved on subsequent transfer like the parent stocks. Among the remainder, by far the most numerous were the slowgrowing and palmelloid types. These all grew in minimal media to some extent, and rather better in 'complete': but since WT cultures behaved in the same way, this provided no indication of biochemical deficiency, or of changed specific requirements. Thirty-four of these were further tested in seven different (liquid) media, consisting of minimal (BM); BM supplemented by one of the following: acetate, tryptone, yeast extract, nucleic acid hydrolysate, casein hydrolysate; and 'complete'; but no specific nutritional responses were observed. Ten mutants were tested for a requirement of unknown substances diffusing from WT cells, by streaking a suspension of WT on pour plates 
densely seeded with the mutant in question: no halo of satellite colonies was observed in any instance.

A few possible mutants failed to survive, for various reasons, before they could be examined microscopically and culturally. The classification of the remainder could not be entirely objective, owing to the range of variability and of characters considered, so the numbers within the classes given in Table 3 should not be regarded as more than indicative of the types of mutant sensu lato obtained in the course of this survey.

Certain mutants will be described in some further detail below.

Table 3. Summary of mutant types

\begin{tabular}{|c|c|c|}
\hline Character & & $\begin{array}{c}\text { No. of } \\
\text { isolates }\end{array}$ \\
\hline \multicolumn{2}{|c|}{ Slow-growing (not characterized further) } & 65 \\
\hline Palmella with some motile cells & 51 & \\
\hline Palmella without motile cells & $16\}^{5}$ & 67 \\
\hline \multicolumn{2}{|l|}{ Strong tendency to misdivisions* } & 10 \\
\hline \multicolumn{2}{|l|}{ Paralysed, etc.* } & 7 \\
\hline \multicolumn{2}{|l|}{ Palet } & 6 \\
\hline \multicolumn{2}{|l|}{ Intracellular volutin abundant* } & 5 \\
\hline \multicolumn{2}{|l|}{ Cell form variants* } & 3 \\
\hline \multicolumn{2}{|l|}{ Flagellaless* } & $\mathbf{3}$ \\
\hline \multicolumn{2}{|l|}{ Vitamin-dependent* } & 2 \\
\hline \multicolumn{2}{|l|}{ Non-autotrophic* } & 1 \\
\hline \multirow[t]{2}{*}{ Slight differences in colony form } & & 25 \\
\hline & Total & 194 \\
\hline
\end{tabular}

* Examples of these types are dealt with separately in the following sections.

$\dagger$ No marked colour variants-e.g. yellow, brown or white-were found. M. 546 cells are appreciably paler than those of $M$. 236, from which it was derived, but in a magnesiacolumn chromatogram of an acetone extract no significant difference between the two types could be detected.

\section{Mutants exhibiting cell misdivisions}

One may occasionally find in WT cultures twinned cells, evidence of incompletely effected divisions. In old cultures, or in the presence of subinhibitory concentrations of various substances in the medium, such forms may be not uncommon. They have been recorded in various species of Chlamydomonas and in related genera. It is probable that many reported cases of 'gamete fusion', in which the process has been described as being initiated at the posterior ends of the cells (Mitra, 1950), are to be attributed to faulty observations of such misdivision forms (as pointed out by Lerche, 1937).

In the case of such mutants as M. 470, an internal physiological upset results in the frequent occurrence of 'Siamese twins' and other abnormalities even in young cultures; it is always associated with a reduced growth rate (Pl. 1, figs. 2, and 4-6).

\section{470(+). Twinning}

Origin. WT + : ultraviolet, $10 \mathrm{~cm}$., $2 \cdot 5 \mathrm{~min}$. (surface, BM bromthymol blue agar). 
This mutant was originally observed to grow less rapidly than WT, and to produce somewhat less wet colonies on BA. Microscopic examination revealed the presence of a high proportion of misdivided cells (e.g. 5-10\% in young liquid cultures). Apparently longitudinal division, proceeding posteriorly from the papillar region, is frequently arrested before completion. Pairs may be found attached by a narrow bridge between the posterior ends (Pl. 1, figs. 2, 5): pairs with a wider bridge, or an extensive region of coalescence: 'cells' with two pyrenoids, two nuclei, and two papillae separated by a notch of variable depth: and, very rarely, a cell with partially fused papillae and three or four flagella as the only external indications of its double nature (Pl. 1, fig. 3). Often three or four cells may remain together, attached by basal processes: such pseudo-colonies resemble a small Pandorina.

Still more grotesque forms may be found, in which a 'cell' may bear four or more processes of irregular dimensions, each bearing a papilla and a pair of apparently normal flagella. Such monsters presumably arise from a series of consecutive misdivisions.

It was found that this mutant is more sensitive to high concentrations of sodium citrate than is WT, the limiting concentrations for maximum growth being about 0.003 and $0.01 \mathrm{M}$, respectively. Liquid media (BA) containing $0.03 \mathrm{M}$ citrate permit growth, in palmellar form, of WT, while almost completely suppressing that of M. $\mathbf{4 7 0}$.

It appeared possible that the action of the citrate might be that of a chelating agent, binding some metal for which $\mathbf{M} . \mathbf{4 7 0}$ has a high requirement. However, a threefold increase of the trace element mixture used throughout these experiments in no way alleviated citrate inhibition. Furthermore, M. 470 responds to various levels of ethylenediamine tetra-acetate (a strong chelating agent-cf. Hutner \& Provasoli, 1951) in the same way as does WT. The phenomenon of citrate inhibition thus remains unexplained.

M. 470 has been used in mating experiments to introduce the character for twinning $(t)$, which behaves as a single gene mutation. Citrate inhibition does not remain associated with it, and the genetics of this incidental feature have not been followed up.

\section{202(+). Monstrous}

Origin. WT + : ultraviolet, $10 \mathrm{~cm}$., $2 \mathrm{~min}$. (surface, BM neutral red agar).

Although the growth rate of this form is not very much lower than that of WT, some serious disorganization is apparent from the form of the cells. Colonies on agar are dry and rough in appearance: in liquid the growth is flocculent, accumulating on the bottom. Most of the cells are grossly misshapen, occasional inflated spheroid forms reaching $50 \mu$. diam., and the lobed, misdivided thalli may contain a multiplicity of nuclei and pyrenoids (Pl. 1, figs. 4, 6). They are as a rule devoid of flagella (occasionally one or two are borne, these generally being shorter than in WT), and, when motile, the mode of swimming is slow and irregular.

No nutritional requirement or pronounced influence of nutrients in the medium has been observed, other than those also exhibited by WT. 


\section{Paralysed mutants}

Among 132 putative mutants arising from irradiation of WT + cells, three (M. 269, M. 375 and M. 475) were identified as being in some way impaired in their flagellar activity: among a further ninety-four obtained from M. 255 ('volutin'), three more (M. 668, M. 700 and M. 701) were found. These, with a mutant (M. 236) which arose from WT + in an earlier experiment, appear to fall into two groups: (1) Paralysed-in which the flagella of the majority of cells remain extended without beating, or occasionally beat at about 1-2 beats/ sec. (compared with about 12 for WT cells). The tips of the flagella-the distal third-twitch sporadically. The autonomous creeping of flagella (Lewin, R. A., $1952 c$ ) is readily apparent in this type, the cells of which may be drawn forward at about $2 \mu$./sec. (M. 236, M. 269, M. 475, M. 668 and M. 701). (2) 'Lazy' - in which the flagella of most cells beat slowly and irregularly, without effectively propelling the cells through the water (M. 375 and M. 700).

In cultures of both categories, especially in those more than a week old, there are found swimming cells, progressing in a wobbling fashion at a rate slower than that typical of WT. The flagellar beat may be of the order of 4-8 beats/sec., and the rate of progress (c. $25 \mu$./sec.) about half that of the normal cells $(c .60 \mu . / \mathrm{sec}$.). Liquid cultures can be at once distinguished from those of fully active cells, since cells of paralysed and 'Iazy', as well as flagellaless and palmelloid, stocks form bottom deposits, whereas WT and most other mutants produce surface films.

As far as has been determined, these mutant flagellar characters are dependent on single genes: in the cases of M. 236, M. 375 and M. 701 these are not allelic, and appear unlinked (Lewin, R. A., 1952b). It may ultimately be possible to garner biochemical information on such mutants which may throw light on the mechanism of flagellar movement as a general phenomenon. So far, unfortunately, no means has been found by which the condition of paralysis can be 'cured' in genetically paralysed cultures.

The origins of the various mutants affecting flagellar characters are summarized in Table 4.

\section{236(+). Paralysed}

Origin: WT + : ultraviolet, $10 \mathrm{~cm}$., $2.5 \mathrm{~min}$. (surface, BM neutral red agar).

This was the first of the series of paralysed mutants to be observed and investigated: further mutants (M. 275-373, M. 406-437 and M. 521-600) were obtained by irradiation of M. 236 cultures.

All attempts to stimulate normal flagellar activity were abortive: such substances as adenosine triphosphate and acetylcholine, glutathione, glucose, hydrogen peroxide, hypertonic sodium chloride, weak acids and alkalis, etc. were without effect in short-term (10-15 min.) tests. A wide range of dilutions of various WT cell hydrolysates, of glucose $(0 \cdot 125-2 \cdot 0 \%)$ and of an aqueous extract of Crocus sativa stigmas (cf. Moewus, 1943) likewise produced no modification of the paralysis during growth. 
This character serves in ready fashion to distinguish the copulants + from during pairing (Lewin, R. A., 1950 a). When the - partner alone is paralysed, the pairs swim in the fashion normal in WT $+\times \mathbf{W T}-$ matings, since the activity of the + partner is unimpaired. When the reciprocal mating is made, the pair does not swim, though the - partner before copulation was capable of normal activity. When both partners are paralysed, mating is of course

Table 4. Pedigrees of mutants with flagellar aberrations

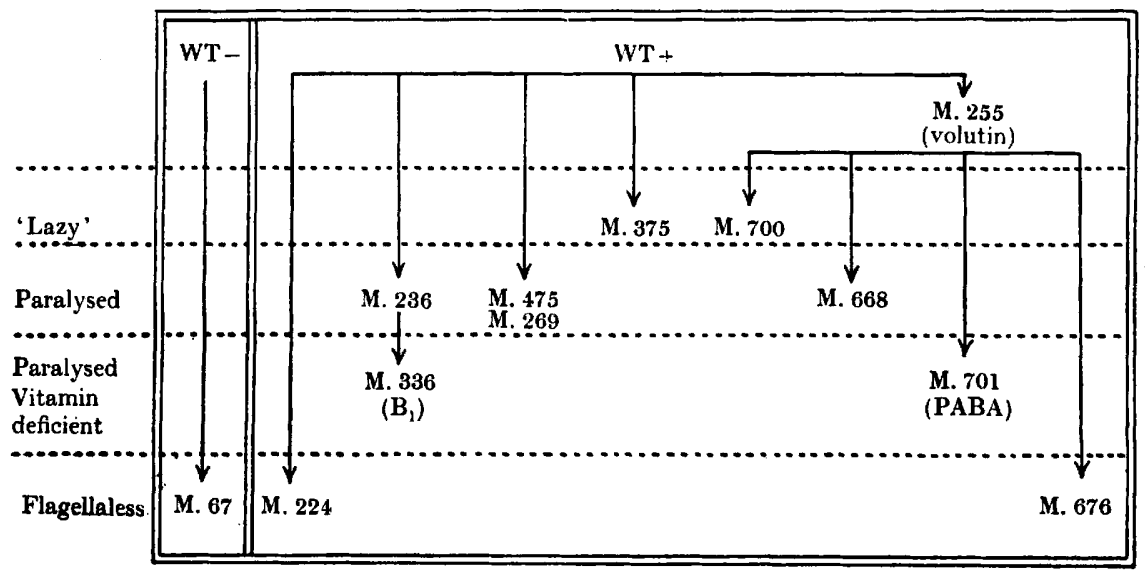

considerably hampered and copulant pairs are sparse, but these again are found to be non-swimming.

Among the mutants derived by further irradiation of M. 236, M. 336 proved to require aneurin for optimal growth. The latter strain, bearing thus a biochemical marker additional to the gene for paralysis, was used in preference to M. 236 for genetic study.

\section{M. $375(+)$. 'Lazy'}

Origin: WT + : ultraviolet, $10 \mathrm{~cm}$., $2 \cdot 5 \mathrm{~min}$. (surface, BM bromthymol blue agar).

Like M. 236, the cells of this mutant accumulate at the bottom in liquid cultures, though in old cultures (e.g. 2 weeks) there is a tendency for the cells to swim through the medium. Their motion is then slow and trembling, however, and unlike that of WT cells.

M. 375 and M. 700 were distinguished from the other mutants of the paralysis series by the greater activity of the non-swimming cells. These perform spasmodic and usually unilateral movements of the flagella, which, beating slowly and asynchronously, fail effectively to propel the cells. Despite this difference of behaviour in young cultures, it is not always possible to distinguish clearly between paralysed and 'lazy' stocks (e.g. in segregates of a cross involving both types). 


\section{255(+). Volutin}

\section{Volutin-rich mutants}

Origin. WT + : ultraviolet, $10 \mathrm{~cm}$., $3 \mathrm{~min}$. (surface, BM bromthymol blue agar).

This mutant, resembling WT in colony form and growth rate on mineral as well as complete media, was revealed by its greater sensitivity to a lowered $\mathrm{pH}$, as a result of which cultures in unbuffered media exhibit a greater tendency to early bleaching. In one experiment, the final $\mathrm{pH}$ after 10 days' growth in $\mathrm{BM}$ was 3.92 (average of five replicates, ranging from 3.83 to 4.00 ), while that of the WT control was $3 \cdot 23$ (five replicates, $3 \cdot 13-3 \cdot 36$ ). Bleaching occurs regularly in BM plates in which the medium is only poured to a depth of $\mathbf{3} \mathrm{mm}$.: where acidification is slower in deeper media of greater volume, the cultures remain green considerably longer.

Microscopically the cells are readily distinguished by the presence of large, refractile globules of 'volutin', which tend to distort the form of many of the smaller cells (Pl. 1, figs. 7, 8). These bodies do not stain with iodine, or with Sudan III or osmic acid, but readily take up neutral red, and presumably correspond with the 'vacuome' described (in Chlamydomonas, etc.) by Hall $\&$ Nigrelli (1931). They exhibit metachromasy when stained with methylene blue; i.e. light transmitted by lightly stained globules tends to be shifted towards the longer wavelengths, so that these bodies take on a lilac or magenta colour.

It was suspected that these 'metachromatic vacuoles' (Hollande, 1942) might consist of metaphosphate (cf. Wiame, 1949). Supporting evidence for this was obtained from a series of cultures in modified BA, in which the amount of phosphate was varied. When phosphate was 'omitted' (traces introduced with the other constituents of the medium and with the inoculum permitting slight growth), no volutin was apparent in either WT or M. 255 cells. At $0.05 \mathrm{~g}$. $\mathrm{K}_{2} \mathrm{HPO}_{4} /$ l., small metachromatic granules appeared in the cytoplasm of most cells in the WT culture, while in M. 255 these were often accompanied or replaced by one or two large globules. In $0 \cdot 15 \mathrm{~g} . \mathrm{K}_{2} \mathrm{HPO}_{4} / \mathrm{l}$. or more, all cells of the mutant contained large globules, ranging up to $5 \mu$. diam. The WT cells contained small granules not exceeding $1 \cdot 5 \mu$. diam.; they appeared in some numbers, about twenty to thirty typically being clustered in the region of the nucleus (cf. Strehlow, 1928-9). In older cultures of WT, however, they may be larger (cf. Czurda, 1935, fig. 6c).

This character of M. 255-the production of large volutin globules-proved to be a convenient marker. When cells so labelled were mated with normal gametes, it was possible to distinguish the partners, plus from minus, and in this way to elucidate differences in their behaviour (Lewin, R. A., 1950a). For many hours, up to the time of nuclear fusion and zygote formation, the respective cytoplasms of the two copulants could be recognized in this way.

By irradiation of M. 255 cultures, a series of mutants was obtained, including a range of variants similar to those obtained from WT cells, but marked with the volutin character. 
From matings with WT, this character was introduced into cells of minus mating-type.

Zygospores homozygous for this character developed regularly and were normal in appearance.

\section{389(+). Volutin}

Origin. WT + : ultraviolet, $10 \mathrm{~cm} ., 2.5 \mathrm{~min}$. (surface, BM bromthymol blue agar).

In all essential respects this mutant resembles $M$. 255. When M. 389 was crossed with Z. 717/2, bearing the volutin gene from M. 255, zygotes were produced which proved to give extremely low and irregular germination after the dark treatment found successful for WT crosses (Lewin, R. A., 1949). This may be attributed to the same alteration in metabolism as that responsible for the formation of large volutin globules in the haplophase. On analysis of one zygote obtained from this cross, a clone without the volutin character was obtained, indicating that different loci are involved.

\section{478. $W(+)$. Spherical}

\section{Cell-form variants}

Origin. WT + : ultraviolet, $10 \mathrm{~cm} ., 2 \cdot 5 \mathrm{~min}$. (surface, BM bromthymol blue agar).

This differs from WT not only in its slower growth, but also in cell form, which is characteristically spherical. The cells are small, usually only $4-6 \mu$. diam., and progress in a slow, jerky fashion: in liquid culture they accumulate on the floor of the vessel, comparatively little surface growth being formed.

\section{67(-). Flagellaless}

\section{Flagellaless mutants}

Origin. WT - : ultraviolet, $15 \mathrm{~cm} ., 5 \mathrm{~min}$. (layering).

The growth rate of this mutant approaches that of WT in mineral media. On agar, the colonies appear less smoothly lenticular: in liquid, the cells remain at the bottom of the vessel. The cells are completely devoid of flagella at all stages and in all media tested. Specifically it"may be noted that no effect was produced either by glucose or by aqueous extracts of Crocus sativa stigmas, used in a wide range of concentrations (cf. Moewus, 1943). In other ways there appear to be no marked differences from WT, though there is a stronger tendency for the formation of palmellar clumps. Gamete-like cells-identified by their size and shape and by their mode of development-are produced: lacking flagella, however, they are incapable of copulating.

\section{Vitamin-dependent mutants (Lewin, R. A., 1950b)}

\section{701(+). PABA-less (paralysed)}

Origin. M. 255 : ultraviolet, $10 \mathrm{~cm}$., $3 \mathrm{~min}$. (surface, $B M$ neutral red agar).

The stock from which this mutant was obtained had already a genetic marker-'volutin'. When M. 701 was isolated, it was found to differ from 
M. 255 in two features: (1) paralysed flagella, like those of M. 236, (2) a partial requirement for exogenous $p$-aminobenzoic acid (PABA), in the absence of which growth is considerably retarded. At least three possible hypotheses were entertained to account for this double change: $(a)$ the two altered phenomena might be manifestations of a single mutated gene; $(b)$ a small deletion of a chromosome, bearing genes affecting motility and vitamin synthesis, may have occurred; $(c)$ two independent mutations had taken place simultaneously.

But augmenting the PABA in the medium did not induce motility: and motile cells of M. 255 were not paralysed by any concentration of sulphanilamide tested, which might be expected to interfere with PABA metabolism. These facts seemed to militate against possibility (a) above.

The matter was clinched by mating experiments in which PABA-requirement segregated in normal fashion from paralysis, both behaving as single genes, apparently unlinked (Lewin, R. A., 1952b). The simultaneous occurrence of these two mutations may be considered less surprising when it is realized that eight mutants of the paralysed series appeared among 226 putatives tested from surface irradiations, of which eighty-one maintained, in subculture, recognizable differences from the parent stock. There might then be a $10 \%$ expectation of a biochemical deficiency mutation arising at the same time as an impairment of the flagellar mechanism.

Like the PABA-requiring strain of Neurospora investigated by Tatum \& Beadle (1942), M. 701 was not stimulated by benzoic acid, but growth could be promoted by aniline. $100 \mathrm{mg}$./ l. completely inhibited growth of this mutant; in $25 \mathrm{mg}$. $/$. the growth rate approached the maximum obtainable in PABA media; while there was some response to concentrations as low as $1 \mathrm{mg}$./l. of aniline. The fact that the relative efficiency of aniline was of the order of $1: 100$ (cf. the value $1: 14,000$, given by Tatum \& Beadle for the Neurospora mutant) indicated that aniline probably does not lie on the direct path of PABA synthesis. Sulphanilamide inhibition of WT cultures was reversed by PABA but not by aniline-further evidence against the possibility that PABA synthesis normally passes through aniline as an intermediate.

M. 336(+). Thiamin-less (paralysed)

Origin. M. 236 : ultraviolet, $10 \mathrm{~cm}$., $2 \cdot 5 \mathrm{~min}$. (surface, BM bromthymol blue agar).

This mutant arose from a paralysed stock, and further differed from WT in a biochemical feature. Tested in BATY and in BA, M. 336 was found to grow extremely poorly in the latter medium. It responded particularly well to additions of yeast extract and of a synthetic vitamin mixture: further tests in BA to which single vitamins had been added showed that such a stimulation could be produced by aneurin (thiamin) alone.

In media supplemented by aneurin, the growth rate approaches that of M. 236; in the absence of this factor, growth is very slow, though in both BA and BPM the cells multiply eventually. (Cf. two out of the four aneurin-less mutants of Neurospora described by Tatum \& Bell (1946), in which the requirement was found not to be absolute.) At $50 \mathrm{mg}$. aneurin/l. there is 
some inhibition, growth proceeding maximally at levels between 0.001 and $0.1 \mathrm{mg} . / \mathrm{l}$., and an appreciable response being obtained to concentrations as low as $0.0001 \mathrm{mg}$./l. (i.e. $4 \times 10^{-10} \mathrm{M}$ ). Lwoff $(1947)$ reported that the lower threshold for aneurin in Polytomella caeca is of the order of $2 \times 10^{-10} \mathrm{M}$.

The vitamin requirement for such a mutant might be satisfied by the free aneurin present in such natural habitats as lake water, where it may reach 0.001 mg./l. (Hutchinson, 1943) or in soil, where it must be considerably higher. Both van Overbeek (1940) and Ondratschek (1940) reported a marked stimulation of Sphaerella-'wild' species-by aneurin, while the latter author published an account of similar phenomena in six Chlamydomonas species. (This work of Ondratschek is open to considerable criticism, however: see Lwoff, 1947.) Thus a mutation of this sort might be under only slight adverse selection in nature.

The thiazole and pyrimidine moieties of aneurin (4-methyl-5-beta-hydroxyethyl thiazole: 2-methyl-5-ethoxymethyl-6-aminopyrimidine) were tested for their ability to replace the complete thiamin molecule. The results are summarized in Table 5 .

Table 5. Response of $M .336$ in $B \boldsymbol{A}$ medium to moieties of aneurin

\begin{tabular}{lc}
\multicolumn{1}{c}{ Supplement } & Growth \\
Control & - \\
0.1 mg./l. thiazole & - \\
0.1 mg./l. pyrimidine & + \\
Thiazole + pyrimidine & + \\
$\mathbf{0 \cdot 2}$ mg./. aneurin & +
\end{tabular}

These results indicate that the organism retains unimpaired the abilities to synthesize thiazole and to couple it with pyrimidine, but its synthetic ability towards pyrimidine is reduced. It may be noted that Polytoma caeca requires additions to the culture medium of both constituents of the aneurin molecule, while Polytomella ocellatum requires the thiazole moiety alone. M. 336, in its synthetic disability, resembles Euglena gracilis (Lwoff, 1947).

\section{Mutant roith impaired photosynthetic system}

\section{151(-). Non-autotrophic}

Origin. WT - : ultraviolet, $15 \mathrm{~cm} ., 2 \mathrm{~min}$. (layering).

This strain was found to be incapable of appreciable growth in mineral liquid media, where it produces small granules of palmella which only become visible to the naked eye 3-4 weeks after inoculation. On media containing an organic carbon-source, however, rates of growth of M. 151 approach those of WT.

A number of compounds were tested for their ability to support growth. Among carboxylic acids (0.007 $\mathrm{M}$, adjusted in $\mathrm{BM}$ to $\mathrm{pH} 7 \cdot 1)$, citrate provides a very suitable source of organic carbon, while good growth can also be obtained with fumarate, succinate, pyruvate or malate: growth with acetate is poorer. Glucose and glycerol also provide suitable carbon sources for growth. 
This mutant has been shown to exhibit the Hill reaction (Lewin, J. C., unpubl.), and to carry out photosynthesis when supplied with $5 \% \mathrm{CO}_{2}$ (Frenkel, A., 1949, private communication). Further studies on its metabolism will be published elsewhere.

Cultures of M. 151 were found to grow in mineral media supplemented with yeast extract $(0 \cdot 1 \%)$, but this effect could not be reproduced by the addition of pure vitamins, either singly or in combination, and it was deduced that the extract merely provided a source of organic carbon.

The pigments of M. 151 and WT cells were compared on a sucrose chromatographic column, using petrol ether as solvent. No significant differences were observed.

For the production of active gamete suspensions, it was found that cultures grown on acetate (BA) were inferior to those on citrate media, and the best results were obtained when both sodium acetate and sodium citrate $(0.1 \%$ of each) were incorporated into the medium.

M. 151 has been carried through a number of crosses, in which the mutant character $(n)$ has behaved as a single Mendelian factor. There is a considerable reduction of viability in segregates bearing this gene, rendering the genetic data for this character very incomplete (Lewin, R. A., 1952b).

As a result of crosses, the character $(n)$ has been transferred to stocks of plus mating-type, and zygotes homozygous for $n$ have been obtained. They require a longer period of illumination for maturation, but ultimately develop on mineral agar, and then appear indistinguishable in size and sculpturing from WT zygospores. This is of some interest, since a $\mathbf{3 0}$-fold increase in bulk is thus effected by the diploid cell in the absence of an organic carbon-source; this corresponds to about five generations of vegetative cells in the haploid condition.

The foregoing data are part of a dissertation presented to the faculty of the Graduate School of Yale University in candidacy for the degree of Doctor of Philosophy. The work was carried out during tenure of Sheffield and H. D. Hooker Fellowships between 1948 and 1950. The author is particularly indebted to $\mathrm{S}$. E. Reaume for many valuable ideas and criticisms in this phase of the investigation; and to various members of the Osborn Botanical Laboratory, to S. H. Hutner and L. Provasoli of the Haskins Laboratories, and to J. C. Lewin.

The samples of thiazole and pyrimidine were obtained through the kindness of Dr J. C. Woodruff of Merck and Co.

\section{REFERENCES}

Burkholder, P. R. \& Nickeld, L. G. (1949). Atypical growth of plants. I. Culture of virus tumors of Rumex on nutrient agar. Bot. Gaz. 110, 426.

Czurda, V. (1935). Über die Variabilität von Chlamydomonas eugametos Moewus. Beih. bot. Zbl. $53 \mathrm{~A}, 133$.

Gerloff, J. (1940). Beiträge zur Kenntnis der Variabilität und Systematik der Gattung Chlamydomonas. Arch. Protistenk. 94, 311.

Hall, R. P.\& NigReldi, R. F. (1931). The vacuome of the flagellate Chlamydomonas. J. Morph. 51, 527.

Hollande, A. (1942). Étude cytologique et biologique de quelques flagellés libres: Volvocales, etc. Thesis, Faculté des Sciences, Univ. Paris.

Hurchinson, G. E. (1943). Thiamin in lake waters and aquatic organisms. Arch. Biochem. 2, 143.

G M VI $3 \& 4$ 
Hutner, S. H. \& Provasoli, L. (1951). The Phytoflagellates. In Biochemistry and Physiology of Protozoa. Ed. Lwoff, A.; New York: Academic Press Inc.

LEA, D. E. (1947). Actions of Radiations on Living Cells. Cambridge University Press.

LederberG, J. \& Tatum, E. L. (1946). Detection of biochemical mutants of micro-organisms. J. biol. Chem. 165, 381.

LERCHE, W. (1937). Untersuchungen über Entwicklung und Fortpflanzung in der Gattung Dunaliella. Arch. Protistenk. 88, 236.

LEwin, J. C. (1950). Obligate autotrophy in Chlamydomonas moevousii Gerloff. Science, 112, 652.

Lewin, R. A. (1949). Germination of zygospores in Chlamydomonas. Nature, Lond. $164,543$.

LEwin, R. A. (1950a). Gamete behaviour in Chlamydomonas. Nature, Lond.166, 76.

Lewin, R. A. (1950b). Induced vitamin-requiring mutants of Chlamydomonas. Nature, Lond. 166, 196.

Lewin, R. A. (1952a). The life-cycle of Chlamydomonas moerousii Gerloff (to be published).

Lewin, R. A. (1952b). The genetics of Chlamydomonas moewusii Gerloff. J. Genet. (in the Press).

Lewin, R. A. (1952c). Studies on the flagella of algae. I. General observations on Chlamydomonas moerousii Gerloff. Biol. Bull. Woodshole (in the Press).

LwoFf, A. (1947). Some aspects of the problem of growth factors for protozoa. Ann. Rev. Microbiol. 1, 101.

Mrtra, A. K. (1950). Two new algae from Indian soils. Ann. Bot., Lond, 14, 457.

Moewus, F. (1940a). Die Analyse von 42 erblichen Eigenschaften der Chlamydomonas eugametos-Gruppe. Z. indukt. Abstamm. u. VererbLehre, 78, 418.

Moewus, F. (1940b). Über Mutationen der Sexual-Gene bei Chlamydomonas. Biol. $\mathrm{Zbl.60,597.}$

Moewus, F. (1943). Geisselbildung und Beweglichkeit bei Chlamydomonas. Naturwissenschaften, 31, 420.

ONDRATSCHEK, K. (1940). Experimentelle Untersuchungen über den Einfluss von Wirkstoffen auf die Vermehrung einigen Mixotropher Algen. Arch. Mikrobiol. $11,89$.

OverBeEK, J. VAN (1940). Traumatic acid and thiamin as growth factors for algae. Proc. nat. Acad. Sci., Wash. 26, 441.

Perkins, D. D. (1949). Biochemical mutants in the smut fungus Ustilago maydis. Genetics, 34, 607.

Smith, G. M. \& Regnery, D. C. (1950). Inheritance of sexuality in Chlamydomonas Reinhardi. Proc. nat. Acad. Sci., Wash. 36, 246.

StremLow, K. (1928-9). Utber die Sexualität einiger Volvocales. Z. Bot. 21, 625.

TATUM, E. L. \& BeAdLe, G. W. (1942). Genetic control of biochemical reactions in Neurospora: an 'aminobenzoicless' mutant. Proc. nat. Acad. Sci., Wash. 28, 234.

TAtum, E. L. \& Bell, T. T. (1946). Neurospora. III. Biosynthesis of thiamin. Amer. J. Bot. 33, 15.

WiAME, J. M. (1949). The occurrence and physiological behaviour of two metaphosphate fractions in yeast. J. biol. Chem. 178, 919 .

\section{EXPLANATION OF PLATE}

(Magnification: figs. 1-6, $\times 1000$; figs. 7, 8, $\times 1500$.)

Fig. 1. Normal, wild-type cell. (Osmic acid; gentian violet.)

Fig. 2. Twinned cells (M. 470). (Osmic acid; gentian violet.)

Fig. 3. Cell with four flagella (M. 470). (Osmic acid; gentian violet.)

Fig. 4. Lobed cell masses (M. 202). (Osmic acid; gentian violet.)

Fig. 5. Twinned cells (M. 470). (Acid hydrolysis; gentian violet.)

Fig. 6. Lobed cell masses (M. 202). (Acid hydrolysis; gentian violet.)

Figs. 7, 8. Cells with large volutin globules (M. 255). (Methylene blue.)

(Received 28 August 1951) 
Journal of General Microbiology, Vol. 6, Nos. 3 and 4
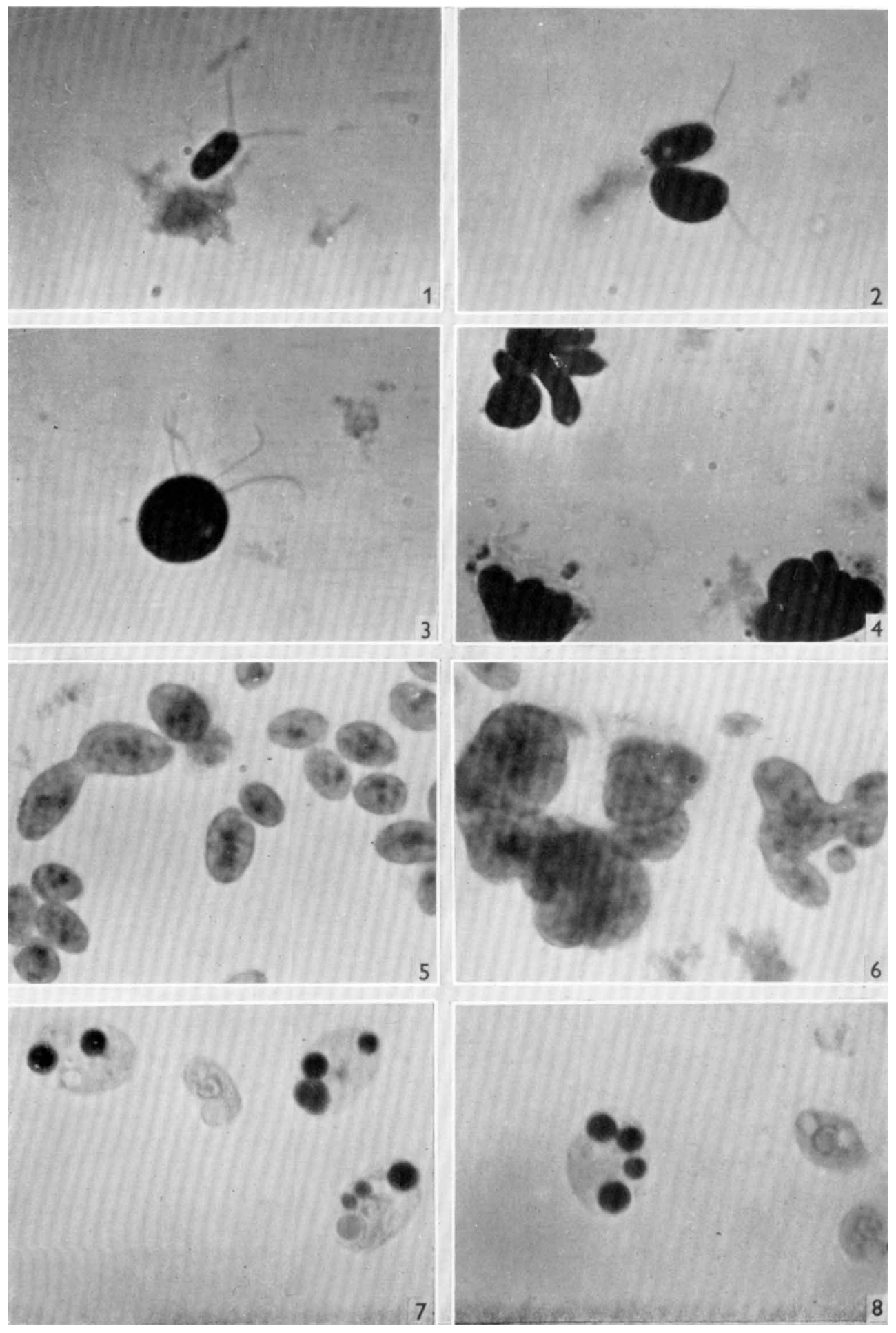

R. A. Lewin-Ultraviolet mutations in Chlamydomonas moewusis. Plate 1 